\title{
Perfil epidemiológico dos casos de leishmaniose tegumentar americana no estado do Pará, Brasil, entre 2008 e 2017
}

\section{Epidemiological profile of American cutaneous leishmaniasis cases in Pará State, Brazil, between 2008 and 2017}

Luciano Sami de Oliveira Abraão' (iD), Bárbara Mendonça Paiva Antônio José2 (iD, Camila Beatriz da Silva Gomes' (iD, Priscila Castilho Nunes² (D), Deivid Ramos dos Santos ${ }^{1}$ (D), Ana Paula Aparecida dos Santos Varela ${ }^{3}$, Caren dos Santos Limaª $^{2}$ (D)

${ }^{1}$ Universidade do Estado do Pará, Belém, Pará, Brasil

2 Universidade Federal do Pará, Belém, Pará, Brasil

${ }^{3}$ Universidade do Estado do Pará, Marabá, Pará, Brasil

\begin{abstract}
RESUMO
OBJETIVO: Descrever o perfil epidemiológico dos casos de leishmaniose tegumentar americana (LTA) no estado do Pará, Brasil, de 2008 a 2017. MATERIAIS E MÉTODOS: Estudo quantitativo e retrospectivo, realizado a partir de dados coletados no Sistema de Informação de Agravos de Notificação sobre os casos notificados de LTA. Os dados foram agrupados segundo as variáveis idade, sexo, raça/cor e município de residência e analisados por meio do qui-quadrado, utilizando o programa BioEstat v5.3, observando o p-valor $<0,05$. Para a confecção do mapa, utilizou-se o TabWin v.4.15. RESULTADOS: Foram notificados 34.609 casos confirmados. A incidência foi de 43,89 casos/ 100.000 habitantes. Houve predominância do sexo masculino $(79,88 \%)$, idade entre 20 a 39 anos $(48,82 \%)$ e pardos $(71,77 \%)$. Registrou-se ocorrência significativa em mulheres, crianças menores de 10 anos de idade e idosos. A forma cutânea foi predominante em $97,39 \%$ dos casos, e 72,19\% evoluíram para cura. CONCLUSÃO: A LTA ainda é um grave problema de saúde pública no Pará, apresentando alta incidência na população. A distribuição espacial dos casos não é homogênea no território paraense, possuindo maior concentração em áreas distantes da capital do estado. Sugere-se a existência de transmissão peri e intradomiciliar relacionada ao acometimento de crianças menores de 10 anos de idade e idosos.
\end{abstract}

Palavras-chave: Leishmaniose Tegumentar Americana; Vigilância Epidemiológica; Distribuição Espacial.

\section{ABSTRACT}

OBJECTIVE: To describe the epidemiological profile of American cutaneous leishmaniasis (ACL) cases in Pará State, Brazil, from 2008 to 2017. MATERIALS AND METHODS: A quantitative and retrospective study was carried out with data from the Information System on Diseases of Compulsory Declaration (SINAN) on notified cases of ATL. The data were grouped according to age, sex, race/color, and municipality of residence. Statistical analyzes were performed using BioEstat v5.3 software, applying the chi-square and p-value $<0.05$. To make the map, TabWin v.4.15 was used. RESULTS: A total of 34,609 confirmed cases were reported. The incidence was 43.89 cases/100,000 inhabitants. There was a predominance of males (79.88\%), aged between 20 to 39 years (48.82\%), and brown-skinned people (71.77\%). There was a significant occurrence in women, children under 10 years old, and elderly people. The cutaneous form was predominant in $97.39 \%$ of the cases, and $72.19 \%$ evolved to cure. CONCLUSION: ACL is still a serious public health problem in Pará, with a high incidence in the population. The spatial distribution of cases is not homogeneous in the State, having a greater concentration in areas distant from the capital, Belém. It is suggested the existence of peri and intra-household transmission related to the incidence of the disease in children under 10 years old and in elderly people.

Keywords: American Cutaneous Leishmaniasis; Epidemiological Monitoring; Spatial Distribution.

\section{Correspondência / Correspondence:}

Deivid Ramos dos Santos

Passagem Maciel, 120. Bairro: Benguí. CEP: 66630-210 - Belém, Pará, Brasil - Tel.: +55 (91) 98234-1393

E-mail: deivid_ramos45@hotmail.com 


\section{INTRODUÇÃO}

A leishmaniose tegumentar americana (LTA) é uma das cinco doenças infectoparasitárias endêmicas de maior relevância para a saúde pública no mundo'. É classificada como doença infecciosa negligenciada, iá que ocorre em países pobres e atinge indivíduos vulneráveis e sem acesso aos bens sociais em saúde. Apresenta ampla distribuição global, e a maioria dos casos ocorrem na África, Ásia e Américas ${ }^{2,3}$, estando presente em mais de 80 países. Quase 90\% dos casos das Américas acontecem no Brasil, Bolívia e Peru ${ }^{1,2}$.

Em 2017, dos 49.959 casos de LTA reportados à Organização Pan-Americana da Saúde (OPAS), o Brasil ocupou o primeiro lugar entre 17 países, por ser responsável por aproximadamente $35 \%$ desses, o que demonstra sua importância como agravo e a necessidade de estudos sobre o mesmo².

No Brasil, a LTA é doença de notificação compulsória, classificada como zoonose de transmissão vetorial, apresentando ampla distribuição territorial e com casos de autoctonia confirmados em todas as unidades federativas, especialmente na Região Norte, onde há maior número de casos e coeficientes médios mais elevados ${ }^{2,4}$. Ademais, a LTA é uma das infecções dermatológicas que merece destaque pelo potencial risco de deformidades cutâneas e reflexos psicológicos, que envolvem diretamente o âmbito social e econômico dos acometidos ${ }^{5,6}$.

Devido à heterogeneidade social e ambiental das macrorregiões do Brasil, ações de combate ao vetor e controle da LTA não têm alcançado plena efetividade?. Diferentes espécies de vetor, reservatórios e agentes etiológicos, característicos de cada região e associados à ação do homem no meio ambiente, contribuem para a manutenção do ciclo de transmissão desse agravo ${ }^{8}$.

Diante disso, estratégias para o devido controle devem ser elaboradas para cada microrregião considerando a especificidade e a necessidade de cada uma, o que, portanto, mostra a importância de se estudar a epidemiologia e o comportamento da transmissão dessa doença localmente.

Dentre os estudos consagrados relacionados à LTA, destaca-se o trabalho da equipe liderada pelo Instituto Evandro Chagas no êxito em descrever conceitos sobre a ecoepidemiologia das leishmanioses, sobretudo no estado do Pará, estado com a maior diversidade de espécies no Brasili2,

Nesse sentido, este estudo teve como objetivo descrever o perfil epidemiológico dos casos de LTA no Pará, entre 2008 e 2017.

\section{MATERIAIS E MÉTODOS}

Estudo quantitativo e retrospectivo, realizado a partir de dados secundários sobre casos notificados de LTA, por município de residência, e registrados no Sistema de Informações de Agravos de Notificação (SINAN). Esses dados foram disponibilizados pela Secretaria de Estado de Saúde Pública do Pará (SESPA), em planilha eletrônica, destacando os eventos ocorridos entre janeiro de 2008 e dezembro de 2017.
As seguintes variáveis foram agrupadas: idade, sexo, raça/cor e município de residência. A análise foi realizada por meio dos testes estatísticos não paramétricos qui-quadrado, para expressar associação entre as variáveis. Utilizou-se o programa BioEstat v5.3, adotando p-valor < 0,05 e 95\% de intervalo de confiança.

Foi construída uma imagem para ilustrar a distribuição dos casos, tendo os municípios que compõem o estado do Pará como unidade de análise, utilizando o programa TabWin v4.15, disponibilizado pelo Departamento de Informática do Sistema Único de Saúde?. O coeficiente de incidência foi obtido pela divisão do número absoluto de casos pela população residente de um mesmo município e multiplicando por 100.0001,2,9. A distribuição foi feita por frequências iguais.

\section{RESULTADOS}

Foram notificados 34.609 casos de LTA no estado do Pará entre 2008 e 2017, com média de 3.461 casos ao ano (Tabela 1). A incidência média no período estudado foi de 43,89 casos para cada 100.000 habitantes, variando de 55,30 em 2014 a 21,87 em 2016, sendo o maior e o menor valor, respectivamente.

Segundo a distribuição espacial dos casos, a maior incidência ocorreu em áreas distantes da região metropolitana (Figura 1).

As características da população acometida estão descritas na tabela 2. Observou-se prevalência do sexo masculino (79,88\%; 27.646). Quanto à escolaridade, $23,94 \%$ (8.287) dos indivíduos possuíam entre a $1^{\mathrm{a}} \mathrm{e}$ a $4^{a}$ série incompleta do Ensino Fundamental e 20,45\% (7.076) informou ter cursado da $5^{a}$ a $8^{a}$ série incompleta do Ensino Fundamental. A faixa etária mais acometida foi a de 20 a 39 anos $(48,82 \% ; 16.897)$, seguida por 40 a 59 anos $(19,88 \%$; 6.881) e 10 a 19 anos (19,45\%; 6.732). Na variável raça/cor, foi observada predominância em pardos $(71,77 \% ; 24.838)$, seguida da cor branca $(13,74 \% ; 4.755)$. $\bigcirc$ grupo de etnia indígena constituiu 1,81\% (625) do universo amostral.

Em relação ao tipo de entrada, 32.719 (94,54\%) casos foram classificados como casos novos e $4,45 \%$ (1.541), recidivas. Quanto ao critério de confirmação, 92,19\% (31.907) foi de cunho clínico-laboratorial. A forma clínica predominante foi a cutânea (33.705). De todos os casos registrados, 72,19\% (24.983) evoluíram para cura; 739 indivíduos abandonaram o tratamento (Tabela 3).

\section{DISCUSSÃO}

Observa-se que a LTA é um problema de saúde pública no Brasill,2,3, sobretudo no estado do Pará que, neste estudo, apresentou incidência média de 43 casos/100.000 habitantes, sendo então classificado como área de ocorrência de alta intensidade, conforme critérios da OPAS ${ }^{1}$. É importante frisar que esse indicador vem diminuindo de forma não linear, o que não é devido à diminuição progressiva do número de casos, mas sim ao aumento da população $0^{8,9}$. 
Tabela 1 - Distribuição dos casos notificados de LTA por ano, porcentagem e incidência, conforme população residente no estado do Pará, Brasil, 2008-2017

\begin{tabular}{ccccc}
\hline Ano & Número de casos & $\%$ & População & Incidência* \\
\hline 2008 & 3.845 & 11,11 & 7.321 .493 & 52,52 \\
2009 & 3.484 & 10,07 & 7.457 .119 & 46,72 \\
2010 & 2.570 & 7,42 & 7.588 .078 & 33,87 \\
2011 & 3.879 & 11,21 & 7.688 .593 & 50,45 \\
2012 & 4.278 & 12,36 & 7.822 .205 & 54,69 \\
2013 & 3.211 & 9,28 & 7.999 .729 & 40,14 \\
2014 & 4.482 & 12,95 & 8.104 .880 & 55,30 \\
2015 & 3.785 & 10,94 & 8.206 .923 & 46,12 \\
2016 & 1.816 & 5,25 & 8.305 .359 & 21,87 \\
2017 & 3.259 & 9,41 & 8.366 .628 & 38,95 \\
\hline Total & 34.609 & 100,00 & 78.861 .007 & 43,89 \\
\hline
\end{tabular}

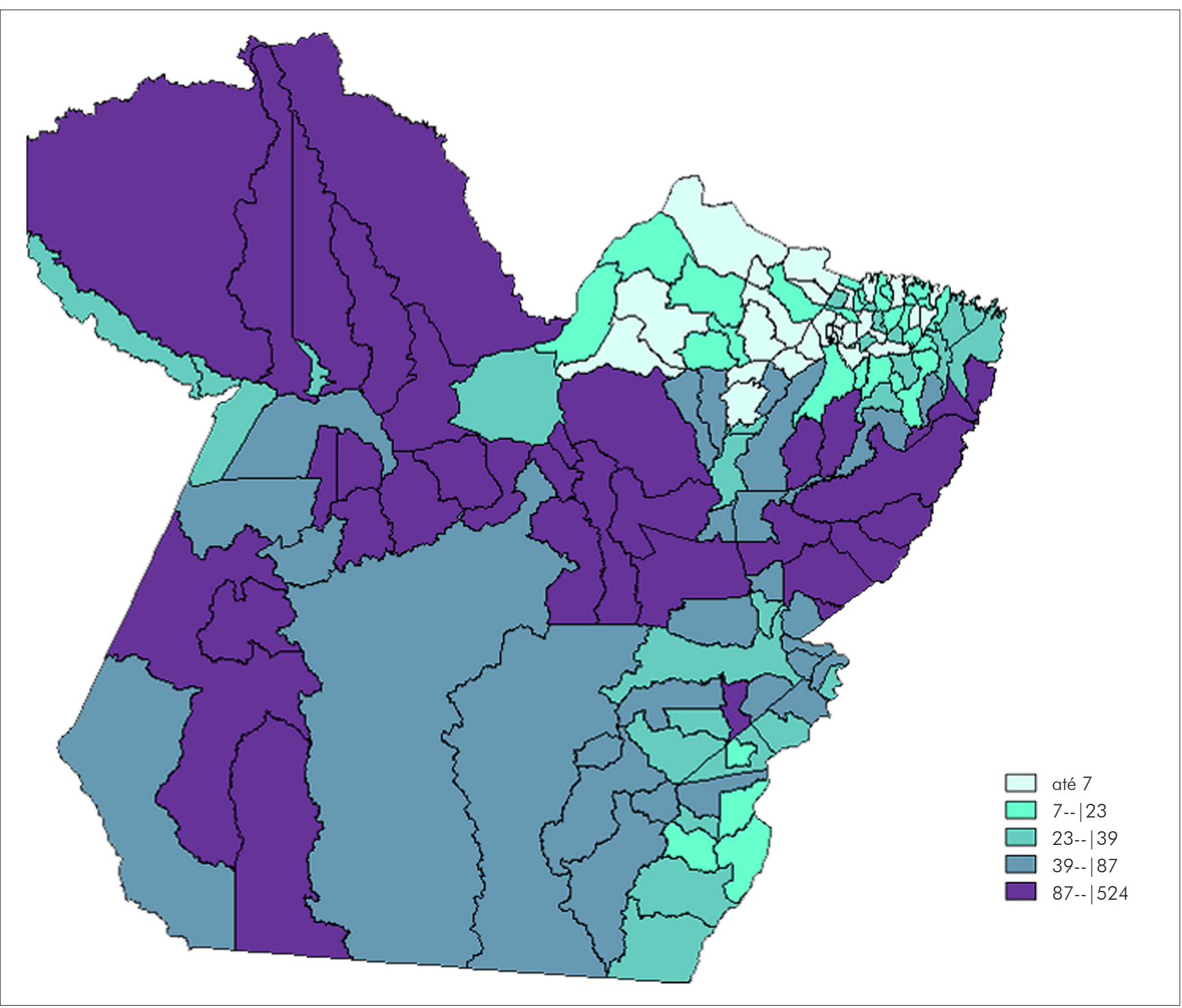

Figura 1 - Coeficiente de incidência dos casos notificados de LTA por município do estado do Pará, Brasil, 2008-2017 
Tabela 2 - Distribuição dos casos notificados de LTA, segundo as variáveis sexo, escolaridade, faixa etária e raça/cor, no estado do Pará, Brasil, 2008-2017

\begin{tabular}{|c|c|c|}
\hline Variáveis & $N=34.609$ & $\%$ \\
\hline \multicolumn{3}{|l|}{ Sexo* } \\
\hline Masculino & 27.646 & 79,88 \\
\hline Feminino & 6.956 & 20,10 \\
\hline Ignorado & 7 & 0,02 \\
\hline \multicolumn{3}{|l|}{ Escolaridade $^{\dagger}$} \\
\hline Analfabeto & 1.396 & 4,03 \\
\hline $1^{a}$ a $4^{a}$ série incompleta do Ensino Fundamental & 8.287 & 23,94 \\
\hline $4^{a}$ série completa do Ensino Fundamental & 4.133 & 11,94 \\
\hline $5^{a}$ a $8^{a}$ série incompleta do Ensino Fundamental & 7.076 & 20,45 \\
\hline Ensino Fundamental completo & 1.765 & 5,10 \\
\hline Ensino Médio incompleto & 1.571 & 4,54 \\
\hline Ensino Médio completo & 1.733 & 5,01 \\
\hline Ensino Superior incompleto & 138 & 0,40 \\
\hline Ensino Superior completo & 231 & 0,67 \\
\hline Não se aplica & 1.590 & 4,59 \\
\hline Ignorado & 6.689 & 19,33 \\
\hline \multicolumn{3}{|l|}{ Faixa etária ${ }^{\ddagger}$} \\
\hline$<10$ & 2.352 & 6,80 \\
\hline 10 a 19 & 6.732 & 19,45 \\
\hline 20 a 39 & 16.897 & 48,82 \\
\hline 40 a 59 & 6.881 & 19,88 \\
\hline 60 a 79 & 1.585 & 4,58 \\
\hline$\geq 80$ & 156 & 0,45 \\
\hline Ignorado & 6 & 0,02 \\
\hline \multicolumn{3}{|l|}{ Raça/Cor } \\
\hline Branca & 4.755 & 13,74 \\
\hline Preta & 3.134 & 9,05 \\
\hline Parda & 24.838 & 71,77 \\
\hline Amarela & 485 & 1,40 \\
\hline Indígena & 625 & 1,81 \\
\hline Ignorado & 772 & 2,23 \\
\hline
\end{tabular}

${ }^{*} p<0,001\left(x^{2} 12371.427\right) ;{ }^{\dagger} p<0,001\left(x^{2} 26957.319\right) ;{ }^{\ddagger} p<0,001\left(x^{2} 43512.751\right) ;{ }^{\S} p<0,001\left(x^{2} 78179.013\right)$. 
Tabela 3 - Distribuição dos casos notificados de LTA, segundo as variáveis tipo de entrada, critérios de confirmação, forma clínica e evolução, no estado do Pará, Brasil, 2008-2017

\begin{tabular}{|c|c|c|}
\hline Variáveis & $N=34.609$ & $\%$ \\
\hline \multicolumn{3}{|l|}{ Tipo de entrada* } \\
\hline Caso novo & 32.719 & 94,54 \\
\hline Recidiva & 1.541 & 4,45 \\
\hline Ignorado & 349 & 1,01 \\
\hline \multicolumn{3}{|l|}{ Critério de confirmação ${ }^{\dagger}$} \\
\hline Clínico-laboratorial & 31.907 & 92,19 \\
\hline Clínico-epidemiológico & 2.702 & 7,81 \\
\hline \multicolumn{3}{|l|}{ Forma clínica ${ }^{\ddagger}$} \\
\hline Cutâneo & 33.705 & 97,39 \\
\hline Mucosa & 896 & 2,59 \\
\hline Ignorado & 8 & 0,02 \\
\hline \multicolumn{3}{|l|}{ Evolução§ } \\
\hline Cura & 24.983 & 72,19 \\
\hline Abandono & 739 & 2,13 \\
\hline Óbito por LTA & 5 & 0,01 \\
\hline Óbito por outra causa & 62 & 0,18 \\
\hline Transferência & 222 & 0,64 \\
\hline Mudança de diagnóstico & 89 & 0,26 \\
\hline Ignorado & 8.509 & 24,59 \\
\hline
\end{tabular}

${ }^{*} p<0,001\left(x^{2} 58404.041\right) ;{ }^{\dagger} p<0,001\left(x^{2} 24644.804\right) ;{ }^{\ddagger} p<0,001\left(x^{2} 64768.936\right) ;{ }^{\S} p<0,001\left(x^{2} 146363.822\right)$.

A distribuição de casos de LTA não foi homogênea no território paraense, sendo menos concentrada em áreas urbanas situadas próximas à capital (Belém), ao norte do estado (Figura 1), semelhante ao encontrado em outros estados ${ }^{10,11,12,13}$. Destaca-se a importância de novos estudos sobre a distribuição espacial desse agravo, delimitando as diferenças entre zonas urbanas e rurais.

Foi encontrada maior taxa de acometimento no sexo masculino em relação ao feminino (proporção de 4:1) e na faixa etária de 20 a 39 anos. Isso sugere um perfil de exposição que se relaciona às atividades laborais. Nas áreas rurais, homens se expõem mais aos vetores responsáveis pela transmissão extradomiciliar ${ }^{12,13,14}$. A ocorrência em mulheres, crianças menores de 10 anos de idade e idosos indica a ocorrência de transmissão peridomiciliar e intradomiciliar $^{13}$. Deve-se destacar o crescente número de mulheres no mercado de trabalho na área rural, possibilitando com que a desproporção entre homens e mulheres acometidos por LTA diminua no decorrer dos anos. No interior do estado, casas de madeira com frestas são comuns, o que propicia a inserção dos flebotomíneos no domicílio durante o dia e facilita a transmissão da Leishmania a animais domésticos, colocando todos em risco, independentemente de idade, atividade profissional e sexo $0^{11,12,13,14}$.

fato da maioria dos casos de LTA ocorrer em indivíduos pardos, deve-se, principalmente, à predominância dessa raça/cor na população do Pará $(65 \%)^{15}$.

Apenas 72,19\% (24.983) dos casos evoluíram para cura, o que pode ser explicado pela condução inadequada dos registros dos pacientes durante 0 tratamento, visto que 24,59\% (8.509) não continham a informação sobre o desfecho final do tratamento.

Este estudo apresenta como limitação o uso de dados secundários, tendo em vista a subnotificação e o preenchimento inadequado das fichas de notificação ${ }^{16}$, 
fato constatado pelo número considerável de variáveis ignoradas (escolaridade, 19,33\%; e evolução da doença, 24,59\%), demonstrando a necessidade de melhorar seu preenchimento por parte dos profissionais.

\section{CONCLUSÃO}

A LTA apresentou alta incidência no Pará no período analisado, acometendo, principalmente, o sexo masculino, pardos e a faixa etária de 20 a 39 anos, estando, dessa forma, intrinsecamente relacionada às atividades laborais dessa população. Sugere-se a existência de transmissão peri e intradomiciliar relacionada ao acometimento de crianças e idosos. A distribuição espacial dos casos não foi homogênea, possuindo maior concentração em áreas distantes da capital do estado.

\section{CONFLITOS DE INTERESSES}

Não houve conflito de interesses no presente estudo.

\section{CONTRIBUIÇÃO DOS AUTORES}

LSOA, BMPAJ, CBSG e PCN contribuíram na obtenção dos dados, revisão e interpretação dos resultados; DRS, APASV e CSL contribuíram na concepção do trabalho, aquisição, análise e interpretação de dados; e CSL contribuiu na redação e revisão crítica do conteúdo.

\section{REFERÊNCIAS}

1 Pan American Health Organization. Leishmaniasis. Epidemiological report of the Americas. Washington: PAHO; 2019 Mar. (Leishmaniasis report; 7).

2 Ministério da Saúde (BR). Secretaria de Vigilância em Saúde. Departamento de Vigilância das Doenças Transmissíveis. Manual de vigilância da leishmaniose tegumentar. Brasília: Ministério da Saúde; 2017.

3 Fahrion A, Gasimov E, Joseph S, Grout L, Allan M, Postigo JR. Surveillance of leishmaniasis in the WHO European Region. Rev Epidemiol Sante Publique. 2018 Jul;66 Suppl 5:S394.

4 Temponi AOD, Brito MG, Ferraz ML, Diniz SA, Silva MX, Cunha TN. Ocorrência de casos de leishmaniose tegumentar americana: uma análise multivariada dos circuitos espaciais de produção, Minas Gerais, Brasil, 2007 a 2011. Cad Saude Publica. 2018;34(2):e00165716.

5 Rocha TJM, Barbosa ACA, Santana, EPC, Calheiros CML. Aspectos epidemiológicos dos casos humanos confirmados de leishmaniose tegumentar americana no Estado de Alagoas, Brasil. Rev Pan-Amaz Saude. $2015 \mathrm{dez} ; 6(4): 49-54$.

6 Carneiro FRO, Amin GA, Cruz LBP, Daher BA. Urban American cutaneous leishmaniasis. An Bras Dermatol. 2018 Jan-Feb;93(1):156-8.

7 Araújo AR, Portela NC, Feitosa APS, Silva OA, Ximenes RAA, Alves LC, et al. Risk factors associated with American cutaneous leishmaniasis in an endemic area of Brazil. Rev Inst Med Trop S Paulo. 2016;58:86.

8 Silva APO, Miranda DEO, Santos MAB, Guerra NR, Marques SR, Alves LC, et al. Phlebotomines in an area endemic for American cutaneous leishmaniasis in northeastern coast of Brazil. Rev Bras Parasitol Vet. 2017 Jul-Sep;26(3):280-4. 10.1590/ s1984-29612017038.
9 Ministério da Saúde (BR). Departamento de Informática do SUS. Informações de Saúde. Informações epidemiológicas e morbidade, doenças e agravos de notificação: leishmaniose tegumentar americana [Internet]. Brasília: Ministério da Saúde; 2018 [citado 2020 mar 12]. Disponível em: http://www2.datasus.gov.br/DATASUS/index. php? area $=02$.

10 Nasser JT, Donalisio MR, Vasconcelos CH. Distribuição espacial dos casos de leishmaniose tegumentar americana no município de Campinas, Estado de São Paulo, no período de 1992 a 2003. Rev Soc Bras Med Trop. 2009 mai-jun;42(3):309-14.

11 Oliveira RZ, Oliveira LZ, Lima MVN, Lima AP, Lima RB, Silva DG, et al. Leishmaniose tegumentar americana no município de Jussara, estado do Paraná, Brasil: série histórica de 21 anos. Rev Saude Publica Parana. 2016 dez; 17(2):59-65.

12 Padilha BG, Albuquerque PV, Pedrosa FA. Indicadores epidemiológicos da leishmaniose tegumentar americana, no período de 1999 a 2008, no Estado de Alagoas, Brasil. Rev Pan-Amaz Saude. 2010 set; 1(3):95-102.

13 Santos GM. Características epidemiológicas da leishmaniose tegumentar americana em um estado do nordeste brasileiro. Arch Health Invest. 2018;7(3):103-7.

14 Guerra JAO, Barbosa MGV, Loureiro ACSP, Coelho CP, Rosa GG, Coelho LIACR. Leishmaniose tegumentar americana em crianças: aspectos epidemiológicos de casos atendidos em Manaus, Amazonas, Brasil. Cad Saude Publica. 2007 set;23(9):2215-23.

15 Instituto Brasileiro de Geografia e Estatística. População residente, segundo a situação do domicílio e condição de indígena - Brasil 1991/2010 [Internet]. Rio de Janeiro: IBGE; 2010 [citado 2020 mar 12]. Disponível em: http:// indigenas.ibge.gov.br/graficos-e-tabelas-2.html. 
16 Nascimento APC, Alves JB, Cardoso VSMM, Brito WI. Aspectos epidemiológicos da leishmaniose tegumentar americana no município de Primavera do Leste, Mato Grosso, MT, Brasil. Saude Coletiva. $2011 ; 8(53): 210-4$.

Recebido em / Received: 13/4/2020 Aceito em / Accepted: 26/8/2020 